Mal J Nutr 26(1): 077-084, 2020

\title{
Effect of Morus alba (white mulberry) leaf on HbA1c of patients with type II diabetes mellitus
}

\author{
Zoha Sohail*, Nighat Bhatty, Saima Naz, Amna Iram \& Saghir Ahmad Jafri \\ Department of Clinical Nutrition, NUR International University Lahore, Pakistan
}

\begin{abstract}
Introduction: Diabetes mellitus is a wide spread metabolic disorder characterized by hyperglycemia. In Pakistan, many traditional or medicinal plants are being used to treat ailments or disorders, both in children and adults. To date, there has been no research study done to investigate the effect of Morus alba (white mulberry) leaves on blood glucose levels of individuals with type II diabetes mellitus in Pakistan. The present study was conducted to determine the effect of Morus alba (white mulberry) leaf powder on glycated haemoglobin (HbA1c) of patients with type II diabetes mellitus. Methods: The study design of this study was a randomised controlled trial. Eighty patients with type II diabetes mellitus were randomly selected from the Fatima Memorial Hospital and were equally divided into two groups - control group and experimental group. Patients in the control group were asked to follow their regular hypoglycaemic medications, while patients in the experimental group were administered with 500mg of Morus alba leaf tablet twice a day, 15 minutes before breakfast and dinner, along with their regular hypoglycaemic medications. $\mathrm{HbA1c}$ of patients in both groups were assessed on day zero before the study and on the ninetieth day at study completion. Results: HbA1c of patients in the control group at baseline was $8.92 \%$ and $8.91 \%$ at final, whereas HbA1c of patients in the experimental group at baseline was $9.13 \%$ and $8.59 \%$ at final. Conclusion: The results of this study concluded that Morus alba leaves had a significant effect in lowering high blood sugar levels.
\end{abstract}

Keywords: Deoxynojirimycin-1, diabetes mellitus, Morus alba, HbA1c

\section{INTRODUCTION}

Diabetes mellitus is a set of metabolic diseases characterised by hyperglycaemia. It is resultant of flaws in insulin secretion, insulin action, or both. Major clinical signs and symptoms of this disease are polyuria, polydipsia, weight loss, delayed wound healing and numbness in extremities. Subjects with glycated haemoglobin (haemoglobin A1c, HbA1c) of $<5.7 \%$ have normal blood glucose levels, while pre-diabetics have $\mathrm{HbA} 1 \mathrm{c}$ of between $5.7 \%-6.4 \%$ and diabetics have $\mathrm{HbA} 1 \mathrm{c}>6.5 \%$ (ADA, 2018).

Many factors are responsible for the increase in the prevalence of diabetes mellitus among different regions of the world. Some factors are urbanisation, harmful eating practices and sedentary lifestyle. A study was conducted in the different provinces of Pakistan to

\footnotetext{
*Corresponding author: Zoha Sohail Department of Clinical Nutrition, NUR International University, Lahore, Pakistan Tel: 0302-8450915; E-mail: zoha1sohail@gmail.com doi: https://doi.org/10.31246/mjn-2019-0055
} 
investigate the prevalence of diabetes mellitus. The duration of the study was from April 2017 to November 2017. Subjects with factors that would affect $\mathrm{HbA} 1 \mathrm{c}$ readings were excluded from the study. These factors included subjects with low haemoglobin ( $\mathrm{Hb})$ levels, with renal and liver disorders, had recently done blood transfusion, used erythropoietin and aged above 20 years. Investigators in the said study had used the American Diabetic Association's criteria for HbA1c. Their results concluded that in Pakistan, $16.98 \%$ were diabetics and $10.91 \%$ were pre-diabetics (Aamir et al., 2019).

A metabolic disorder like diabetes mellitus is a worldwide, global and epidemic disease. It is caused by the decrease in insulin production or decreased uptake due to increased resistance of the cells. Rather than managing this disorder via allopathic medicines, other variables or mediums have been investigated or identified for its management. About $80 \%$ of the people residing in developing countries use medicinal plants for their prime health care and have more trust towards traditional or herbal medications. As western medications or allopathic medications are too costly, therefore traditional medical therapies are often more preferred. Many medicinal plants and traditional medical therapies are undeniably effective (Sarangzai, Ahmed \& Laghari, 2013).

Chae et al. (2003) reported that in Pakistan, a number of medicinal plants are being used for curing and treating diseases. In the plant kingdom, there are many varieties and species of plants available in different regions that are testified for managing high blood glucose values. A research article reported that in India, about sixty five plants are present that work against diabetes mellitus. Among these, Morus alba is a plant that works for the prevention of this disorder
(Mukherjee et al., 2006). Morus alba, also known as Morus Indica, a white mulberry tree is being used for its medicinal properties to cure diabetes mellitus as it possesses an anti-diabetic effect. Functional compounds that are present in its leaves are 1-deoxynojirimycin (DNJ), gamma-aminobutyric acid (GABA) and flavonoids.

Other than Morus alba leaves, many anti-diabetic extracts, anti-diabetic leaves and anti-diabetic seeds have been investigated to lower high blood sugar levels. The extracts of Psidium guajava L., Ficus bengalensis L., Aloe vera Nill, Momordica charantia L., Allium cepa L. and Cajanus cajan have also been found to be helpful for diabetics. The leaves of Zizyphus jujuba Mill and Dodonaea viscosa $L$. have been suggested for people with diabetes. The seeds of Trigonella foenum-graecum $L$. and Withania coagulens $L$. have also been recommended to be consumed by diabetics (Ahmad et al., 2009).

Morus alba leaves had been used traditionally as anti-inflammatory and anti-hyperglycaemic agents (Yatsunami et al., 2003). Human-based studies showed that oral intakes of $0.8 \mathrm{~g}$ and 1.2g Morus alba leaf powder significantly reduced elevated blood glucose levels (Kimura et al., 2007). The content of DNJ present in Morus alba leaf is $1.622 \mathrm{mg} / \mathrm{g}$ (Liu \& Zhu, 2006). A review study was conducted to explore the plants or herbs with medicinal potentials, especially the ones which would have anti glycated properties. Among the many plants reviewed in this study, Morus alba leaves were jotted for its medicinal properties against hyperglycaemia. Leaves of this plant works against hyperglycaemia in such a way that they impede the formation of advanced glycation end products (AGEs). Untreated diabetes for long periods of time will ultimately enhance the production of AGEs in the body, which will further enhance the 
formation of reactive oxygen species (ROS). ROS has a leading role in causing mutations among genetic structures. Therefore, the combined effects of AGEs and ROS among diabetics can cause a devastating impact. Morus alba leaves have a potential to stop the production of AGEs and along with this, they have antioxidants which stops ROS from being effective (Dil, Ranjkesh \& Goodarzi, 2019).

Among all these compounds, the most significant components of Morus alba leaves are DNJ and polyhydroxylated alkaloids, which possess inhibitory power against the working of the carbohydrate metabolising enzyme, alpha glucosidase. This enzyme resides in the small intestine and is firstly concerned with the hydrolysis of disaccharides in the small intestine, and secondly with the absorption of glucose in the blood vessels, which progresses onwards to the increase in blood glucose levels. DNJ present in Morus alba leaf is a strong natural alpha glucosidase inhibitor that works as an opponent of this enzyme in the small intestine, whereby it slows down the action of alpha glucosidase and ultimately decreases the abrupt spike in blood glucose levels; a positive point for type II diabetics (Yatsunami et al., 2003). Therefore, this is predicted to bring a favourable effect on the suppression of abnormally high blood glucose levels (Nakagawa et al., 2007). Literature showed that DNJ content is higher in young Morus alba leaves that are present in the upper branches of the tree, compared to mature leaves. The content of DNJ among Morus alba leaf varies on the basis of region, position of the branch on the tree, soil situation, 24-hourly cycle and climate of the year (Thakur et al., 2019).

The basic objective behind this study is that in Pakistan, there is no research study that has been conducted to describe the effect of Morus alba. Studies had been conducted to investigate the functions of its fruits, but no focus had been laid on the consumption of its leaves. This plant is available worldwide, including in Pakistan, to serve humanity. Natural alpha glucosidase inhibitors present in its leaves is a miracle against elevated $\mathrm{HbA} 1 \mathrm{c}$ levels. So this study helped to estimate the quantity of Morus alba that is required to manage elevated HbA1c levels.

\section{MATERIALS AND METHODS}

\section{Study design and sampling}

The study design of this study was an open label randomised controlled trial. An ethical review letter numbered FMH06-2018-IRB-475-A was signed from the Institutional Review Board (IRB) of Fatima Memorial Hospital (FMH), Lahore to carry out the study. After getting the IRB letter from FMH, 105 subjects were selected for the study. Among them, 80 met the selection criteria i.e. type II diabetic male and female patients, aged up to 60 years, and taking oral hypoglycaemic medications were included in the study. The patients were then equally allocated to treatment groups using GraphPad.

Patients with type I diabetes, type II diabetes on insulin treatment, diabetic patients with chronic disorders and pregnant or lactating mothers were excluded from the study. CONSORT diagram is shown in Figure 1.

Informed written consent was taken from the subjects. After explaining to patients about the aims and objectives of the study, they were ensured of the confidentially of information. After taking consent, randomisation was done using a systematic sampling technique. One group was allocated conventional treatment and the other group was considered as experimental group. They were then further channeled to the three phases of the study: 


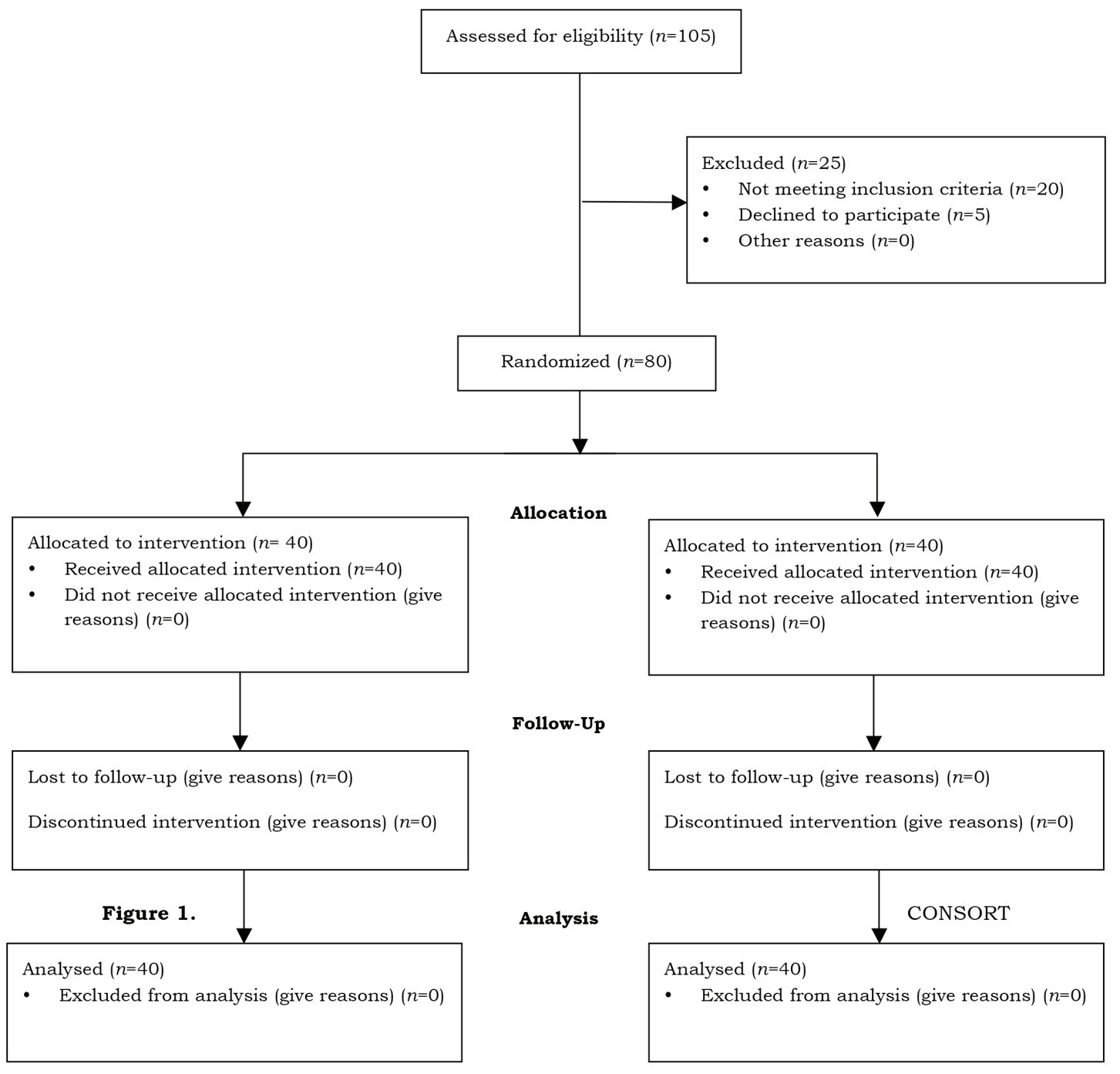

Figure 1. CONSORT diagram for enrollment of subjects

Phase I: Baseline assessment

At baseline, HbA1c levels of patients were assessed according to Bishop, Fody \& Schoeff (2010).

\section{Phase II: Intervention}

The total targeted population was 80 adults with known type II diabetes mellitus taking oral hypoglycaemic drugs. The patients were randomly allocated to treatment groups using GraphPad.

The hypoglycaemic medications of patients in both groups were not altered. Patients in the control group were asked to follow their regular regimens, whereas patients in the experimental group were asked to take 500mg of Morus alba leaf tablets twice a day with water, 15 minutes before breakfast and 
dinner, for a total period of ninety days along with their regular medication. The formulation of the Morus alba leaf tablets was done according to the wet granulation method (Muazu, Abdulwoliyn \& Mohammed, 2013). Substances used during the formulation of Morus alba leaf tablets were lactose monohydrate, corn starch, Avicel-200 and Kollidone K-30. Magnesium stearate was used for coating.

Phase III: Follow-up

After the interventional phase of ninety days, patients' HbA1c readings were measured.

\section{Statistical analysis}

Normality of data regarding $\mathrm{HbA} 1 \mathrm{c}$ was checked using the Shapiro Wilk test for baseline $(p=0.056)$ and for followup $(p=0.068)$. As the data was normally distributed, independent sample $t$-test was applied for testing the difference between groups, while paired sample $t$-test was used to test for baseline and follow-up differences. A $p$-value of $\leq 0.05$ was considered as statistically significant.

\section{RESULTS}

A total of 80 patients were included in the study and they were equally allocated to the experimental and control groups. The duration of diabetes in the experimental group was $5.90 \pm 5.01$ years and in the control group was $8.88 \pm 5.26$ years. The difference between these two groups was statistically significant $(p=0.011)$, as shown in Table 1. Morus alba leaf tablets were uniformed for all the patients to prevent any sort of variation in results. Figure 2 shows the differences among HbA1c of patients in the control group and experimental group. Baseline measurement of HbA1c was taken at the start of the study and a final reading was taken on the ninetieth day.

Figure 2 shows that patients in the control group at baseline i.e. visit 1 had $\mathrm{HbA} 1 \mathrm{c}$ of $8.92 \%$ and at final visit i.e. visit 2 had HbA1c of $8.91 \%$. Results of patients in the experimental group treated with Morus alba leaf showed that at visit 1 , their $\mathrm{HbA} 1 \mathrm{c}$ reading before intervention was $9.13 \%$ and at visit 2 after intervention, $\mathrm{HbA} 1 \mathrm{c}$ reading was $8.59 \%$.

Table 1. Mean, standard deviation and $p$-value of age and duration of disease

\begin{tabular}{lccc}
\hline & Experimental & Control & -value $^{\dagger}$ \\
\hline Mean age of the patients (years) & $53.70 \pm 9.76$ & $53.25 \pm 8.79$ & 0.829 \\
Duration of disease (years) & $5.90 \pm 5.01$ & $8.88 \pm 5.26$ & $0.011^{*}$ \\
\hline
\end{tabular}

Independent sample $t$-test

${ }^{*} p$-value significant at 0.05

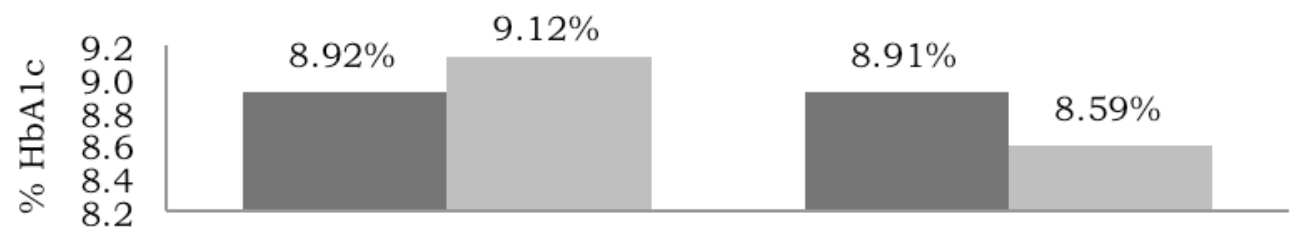

Baseline

Final

- Control Experimental

Figure 2. HbA1c of experimental and control group 
Table 2. Hba1c of diabetic patients in control and experimental group

\begin{tabular}{lll}
\hline Group of patients & $M \pm S D$ & p-value \\
\hline Control group & & 0.559 \\
Baseline & $8.92 \pm 1.36$ & \\
Final & $8.91 \pm 1.26$ & $0.001^{*}$ \\
Experimental group & & \\
Baseline & $9.13 \pm 1.33$ & \\
Final & $8.59 \pm 1.31$ & \\
\hline
\end{tabular}

${ }^{*} p$-value significant at 0.05

Table 2 shows the baseline values taken at the beginning of the study and final values taken at the end of the intervention. The results showed that at baseline, mean $(M)$ and standard deviation $(S D)$ for $\mathrm{HbA} 1 \mathrm{c}$ value of patients in the control group was $8.92 \pm 1.36$, and the final value was $8.91 \pm 1.26$, where they were not statistically significant $(p=0.559) . M$ and $S D$ at baseline for patients in the experimental group was $9.13 \pm 1.33$, whereas the final value was $8.59 \pm 1.3$ which was statistically significant $(p=0.001)$.

\section{DISCUSSION}

The communal methods used for managing hyperglycaemia include inducing insulin or by ingesting hypoglycaemic medications. Many biochemical pathways have been studied to determine the effect of various compounds on hyperglycaemia. Among the investigated compounds, alpha glucosidase inhibitor is a very crucial one in regulating carbohydrate metabolism. This substance is available in the form of medicines and it is naturally available in white mulberry leaves (Ju \& Kim, 2018). Other than this enzyme, white mulberry leaves are composed of proteins, dietary fibre and micro minerals. They provide anti-diabetic, anti-hypertensive, antipyretic and anti-inflammatory properties (Andallu \& Vardacharyulu, 2001).

Insulin therapy provides only insulin and medical therapy only provides specific compound, but white mulberry leaves provide a number of health protecting and health promoting compounds. Its leaves are known to provide anti-oxidative and antiinflammatory properties to serve as free radical scavengers. So, on the basis of all these health benefits, white mulberry leaves can be used to manage hyperglycaemia. Because its constituent compounds will not only cope with high blood sugar level, but also protect an individual from any chronic disorders (Nazari et al., 2013).

Alpha glucosidase is an enzyme accountable for carbohydrate metabolism. When any carbohydratecontaining source is ingested, its activity starts and blood sugar level will begin to rise. Among diabetics, the control of this enzyme's activity is very worthy because the gradual increase in blood sugar level is a more stable condition. Hence, alpha glucosidase inhibitor is a compound that will slow down carbohydrate metabolism and therefore prevent the abrupt rise in blood sugar level (Ju \& Kim, 2018).

A study was conducted on rats induced with diabetes to determine the effect of white mulberry leaf powder on blood glucose levels. The rats were divided into five groups; each group with six rats, and the total time period of this study was 60 days. Group one had normal control rats, group two had normal experimental rats treated with white mulberry leaf powder, group three 
had diabetic controls, group four had diabetic experimental rats to be treated with white mulberry leaf powder and group five had diabetic experimental rats treated with hypoglycaemic medicines. Among these five groups; group one and group three were administered with the standard diet, while group two and group four were administered with an experimental diet incorporated with $25 \%$ level white mulberry leaf powder, and rats in group five received a normal diet along with hypoglycaemic medications. To produce mulberry leaf powder, its leaves were first collected and washed. They were then shade dried for a period of three days and lastly they were ground in an electric mixer to form a powder. Rats were tested for glycosylated haemoglobin and fasting blood glucose levels at first day and at sixtieth day. Results of this study concluded that fasting blood sugar levels among rats treated with mulberry leaf powder were significantly reduced by $50 \%$ when compared with diabetic rats in the control group, whereas there was only $28 \%$ reduction in blood glucose levels in rats treated with hypoglycaemic medications. HbA1c levels were significantly reduced by $30 \%(p<0.01)$ among the rats treated with mulberry leaves, whereas HbA1c levels were decreased by $6 \%$ among rats treated with hypoglycaemic medications (Andallu \& Vardacharyulu, 2001).

Another study was directed to determine the effect of mulberry leaves on diabetic rats. In this study, rats were given $10 \%$ mulberry leaves for a total time period of six weeks. At baseline, the rats were assessed for $\mathrm{HbA} 1 \mathrm{c}$ and the final results showed that $\mathrm{HbA} 1 \mathrm{c}$ values were significantly improved $(p<0.05)$ (Park et al., 2012). Moran 20K and DNJ present in Morus alba leaves have a very affirmative influence on blood sugar levels of people with hyperglycaemia. Other than curing and maintaining blood sugar levels among diabetics, Morus alba leaves, due to its neuroprotective properties, play an impactful role in preventing microvascular and macrovascular complications caused by hyperglycaemia (Butt et al., 2008). During the study, the duration of diabetes seemed to be a contributing factor. In the experimental group, the $M$ and $S D$ for duration of disease was $5.90 \pm 5.01$ and in the control group, the $M$ and $S D$ for duration of disease was 8.88 \pm 5.26 . Therefore, it is suggested that in future studies, the duration of diabetes should be controlled for to reduce cause and effect bias.

\section{CONCLUSION}

The study concluded that Morus alba leaves have a significant effect on hyperglycaemia. End results of the study showed that HbA1c levels of patients in the experimental group were statistically improved $(p=0.001)$, whereas the results of the control group were not significant ( $p=0.559)$. Thus, by consuming Morus alba leaf tablets, high blood sugar levels can be normalised. However, the time and duration of consumption is very important. An ideal time for its consumption is 10-15 minutes before main meals. This study suggested that consumption of $500 \mathrm{mg}$ of Morus alba leaf tablets twice a day could help in the control of blood glucose levels among diabetic patients. It is also recommended that future studies be conducted to investigate the functions of all of its constituents and that studies must be conducted to focus on its form of administration i.e. tablet, powder or extract form.

\section{Acknowledgments}

The authors hereby show sincere appreciation to the subjects and staff of organisation where the study was carried out, for their commitment and cooperation during the total duration of the study. 


\section{Authors' contribution}

ZS, principal investigator hypothesised and set the aims of the study, organised the draft of the manuscript and reviewed the manuscript; NB, led the approval and arrangements of all the official credentials necessary for the study; SN, helped in the drafting and reviewing of the manuscript; AI, led the formulation of statistical methods and formulas; SAJ, led the interpretation of biochemical values obtained from subjects.

\section{Conflict of Interest}

The authors declare that there is no conflict of interest as the study was a self-sponsored project with no financial aid from any donor or organisation.

\section{References}

Aamir AH, Ul-Haq Z, Mahar SA, Qureshi FM, Ahmad I, Jawa A \& Ishtiaq O (2019). Diabetes Prevalence Survey of Pakistan (DPS-PAK): prevalence of type 2 diabetes mellitus and prediabetes using HbA1c: a population-based survey from Pakistan. Br Med J 9(2):e025300.

Ahmad M, Qureshi R, Arshad M, Khan MA \& Zafar $M$ (2009). Traditional herbal remedies used for the treatment of diabetes from district Attock (Pakistan). Pak J Bot 41(6):2777-82.

American Diabetes Association (ADA) (2018). 2. Classification and diagnosis of diabetes: standards of medical care in diabetes-2018. Diabetes Care 41:13-27.

Andallu B \& Vardacharyulu NC (2001). Effect of mulberry leaves on diabetes. Int $J$ Diab Dev Countries 21(147):51.

Bishop ML, Fody EP \& Schoeff LE (2010). Clinical chemistry: principles, techniques, and correlations. Lippincott Williams \& Wilkins, Philadelphia.

Butt MS, Nazir A, Sultan MT \& Schroën K (2008). Morus alba L. nature's functional tonic. Trends Food Sci Technol 19(10):505-512.

Chae JY, Lee JY, Choi SW, Rhee SJ, Hoang IS, Whangbo D \& Kim SY (2003). Analysis of functional components of leaves of different mulberry cultivars. Korean $J$ Food \& Nutr 32(1):15-21.

Dil FA, Ranjkesh Z \& Goodarzi MT (2019). A systematic review of antiglycation medicinal plants. Diabetes \& Metabolic Syndrome: Clinical Research \& Reviews 13(2):1225-1229.

Ju YH \& Kim M (2018). Anti-diabetic Potentials of White Mulberry. Publication HNFE-518, Virginia Cooperative Extension, Virginia Tech, Virginia State University.
Kimura T, Nakagawa K, Kubota H, Kojima Y, Goto Y, Yamagishi K, Oita S, Oikawa S \& Miyazawa $\mathrm{T}$ (2007). Food-grade mulberry powder enriched with 1-deoxynojirimycin suppresses the elevation of postprandial blood glucose in humans. J Agric Food Chem 55(14):5869-5874.

Liu WQ \& Zhu XR (2006). The determination of 1-deoxynojirimycin (DNJ) in three parts of mulberry. Bulletin of Sericulture 4:009.

Muazu J, Abdulwoliyu A \& Mohammed GT (2013). Design, formulation and evaluation of bitter leaf tablets. Int J Pharm Sci Res 4:1789-1795.

Mukherjee PK, Maiti K, Mukherjee K \& Houghton PJ (2006). Leads from Indian medicinal plants with hypoglycemic potentials. $J$ Ethnopharmacol 106(1):1-28.

Nakagawa K, Kubota H, Kimura T, Yamashita S, Tsuzuki T, Oikawa S \& Miyazawa T (2007). Occurrence of orally administered mulberry 1-deoxynojirimycin in rat plasma. J Agric Food Chem 55(22):8928-8933.

Nazari M, Hajizadeh MR, Mahmoodi M, Mirzaei MR \& Hassanshahi G (2013). The regulatory impacts of Morus alba leaf extract on some enzymes involved in glucose metabolism pathways in diabetic rat liver. Clin Lab 59(56):497-504.

Park JH, Lee KW, Sung KS, Kim SS, Cho KD, Lee BH, \& Han CK (2012). Effect of diets with mulberry leaf and Cudrania tricuspidata leaf powder supplements on blood glucose-related biomarkers in streptozotocin-induced diabetic rats. Korean J Food \& Nutr 41(6):766-773.

Sarangzai AM, Ahmed A \& Laghari SK (2013). Traditional uses of some useful medicinal plants of Ziarat District Balochistan, Pakistan. FUUAST J Biol 3(1):101.

Thakur K, Zhang YY, Mocan A, Zhang F, Zhang JG \& Wei ZJ (2019). 1-Deoxynojirimycin, it's potential for management of noncommunicable metabolic diseases. Trends Food Sci Technol 89:88-99.

Yatsunami K, SaitoY, Fukuda E, Onodera S \& Oshigane K (2003). a-Glucosidase inhibitory activity in leaves of some mulberry varieties. Food Sci Technol Res 9(4):392-3. 